\title{
Are the elderly more vulnerable to psychological impact of natural disaster? A population-based survey of adult survivors of the 2008 Sichuan earthquake
}

\author{
Zhaobao Jia', Wenhua Tian*1, Weizhi Liu², Yang Cao³, Jin Yan² and Zhisheng Shun ${ }^{4}$
}

\begin{abstract}
Background: The association between ages and psychological impact of natural disasters has not been well characterized. A population-based study was conducted 15 months after the 2008 Sichuan earthquake to assess whether elderly survivors were more likely to develop posttraumatic stress disorder (PTSD) and general psychiatric morbidity.

Methods: A population-based survey of 327 survivors (152 elders, 175 younger adults) was conducted in severely affected areas by the earthquake, using a multi-stage systematic sampling design.

Results: Compared with the younger adult survivors, the elderly were more likely to have symptoms of PTSD ( $22.5 \%$ vs. $8.0 \%, p=0.001)$ and general psychiatric morbidity ( $42.0 \% \mathrm{vs} .25 .4 \%, p=0.003)$. Risk factors, such as being elderly, having been in serious danger, having lost family members, and having felt guilt concerning one's death or injury were significantly associated with developing PTSD; being elderly, having family members or friends seriously injured, and having felt guilt concerning one's death or injury were significantly associated with developing general psychiatric morbidity. Utilization of mental health services is strongly associated with the decreased risk for developing both of the symptoms.
\end{abstract}

Conclusion: Compared with the younger adults, the elderly survivors were more likely to develop PTSD and general psychiatric morbidity. More mental health services should be distributed to the elderly and groups at particular risk, to ensure their smooth mental health reconstruction after the earthquake.

\section{Background}

Older people are among the most vulnerable populations to the direct impact of natural disasters. Previous studies have proven that physical wellbeing of the elderly is more affected than in younger adults. For instance, a rapid assessment of the health status of people in the affected areas following the Sichuan earthquake suggested that the greatest morbidity was among those over 60 years of age [1]. After Hurricane Charley, at least one older adult's medical condition had worsened in one-third of households that included one or more older adults with a preexisting medical condition [2]. The difference has been

\footnotetext{
* Correspondence: wh_tian@smmu.edu.cn

1 Faculty of Health Service, Second Military Medical University, Shanghai, China Full list of author information is available at the end of the article
}

attributed to decreased sensory awareness (sight, smell, hearing, and touch), physical impairment, chronic health conditions, and socioeconomic limitations experienced by many of the elderly [3].

Previous studies also discussed the psychological impacts of natural disasters, and posttraumatic stress disorder (PTSD) has been one of the focuses [4-6]. PTSD is a severe anxiety disorder that can develop after exposure to any event which results in psychological trauma [7]. It has been found to be the most prevalent type of psychiatric morbidity after disasters, such as earthquake and tsunami [8]. Signs and symptoms of PTSD include re-experiencing original trauma(s), by means of flashbacks or nightmares; avoidance of stimuli associated with the trauma; 
and increased arousal, such as difficulty falling or staying asleep, anger, and hypervigilance [7].

However, conclusions about the psychological impacts of natural disasters on elder and younger adults have been equivocal. In a population-based sample of the 1998 flood victims in China, Liu et al. reported that the elderly were over twice more likely to develop symptoms of PTSD [9], Ticehurst et al. found the elderly to be more distressed than younger adults after an earthquake [10], whereas other studies suggested that the elderly were less susceptible to psychological disorders or were consistent with the symptoms of the general population [11]. For instance, in a study undertaken 18 months after the 1988 earthquake in Armenia, no difference was found on the overall severity of PTSD symptoms between the elderly and younger adults [4], and the results of an epidemiological study after Hurricane Honduran indicated that the elderly survivors were at equal risk for developing PTSD as the younger survivors [12]. The inconsistency in the outcomes has been attributed to the properties of disasters chosen for study as well as methodological differences (e.g., methods of sampling and case detection) [6]. However, socioeconomic contexts may also play an important role in modifying the outcomes. Hence, as prior studies on the issue were mostly performed in developed countries, more information from middle- and low-income settings is needed. To our knowledge, this is the first post-disaster study that has been specially designed to investigate the psychological disparities between elderly and younger adult survivors in the developing world. In addition, we introduced utilization of mental health services as an independent variable and explored its associations with the prevalence of mental health symptoms, which was seldom discussed in previous post-disaster studies.

\section{Methods \\ Setting and design}

On May 12, 2008, a powerful earthquake measuring 8.0 on the Richter Scale struck Sichuan Province, Southwest of China. More than 69,200 people were confirmed dead, more than 374,600 were seriously injured, and more than 17,900 were reported missing [13], making it one of the deadliest natural disasters in history. The earthquake spread about 100,000 square kilometers, left at least 5 million people without housing, and destroyed millions of livestock and a significant amount of agriculture. The official estimates of the direct economic losses were reported at 123.8 billion USD [14].

In this study, we surveyed adult survivors in the earthquake-affected areas using a multi-stage systematic sampling design. To obtain sufficient power to detect differences in outcomes between the elderly and younger adults, we estimated that 266 participants (133 in each arm) were required to detect a relative risk of 2.5 for PTSD symptoms when the prevalence of the outcome in the comparison group is $10 \%$. Estimates of post-disaster PTSD prevalence in East-Asian populations typically vary between $5 \%$ and $30 \%$ [5,6,15-17], and we selected the PTSD prevalence (10\%) of the 1998 Hunan flood victims in China to calculate the sample size due to the similarity in the impact of the disasters as well as the socioeconomic context [16]. We selected $90 \%$ power in the calculation to accommodate the underlying design effect so that 266 participants would provide sufficient power to detect the differences of interest between the two arms. The final sample size was adjusted to 315 subjects (148 elders and 167 younger adults) to account for a potential $10 \%$ and $20 \%$ non-response rate for the elderly and younger adults, respectively (a higher non-response rate was estimated for the younger adults from pilot data, which could be explained by the internal migration in the area) [18].

We randomly selected two of the most severely affected subdistricts from a total of ten [19] (Figure 1). We draw a systematic sample for both elders and younger adults in the available villages with the assistance from the local household registration department. An elder was defined as he or she with at least 60 years of age. When sampling elders, we first calculated a sampling ratio (target number

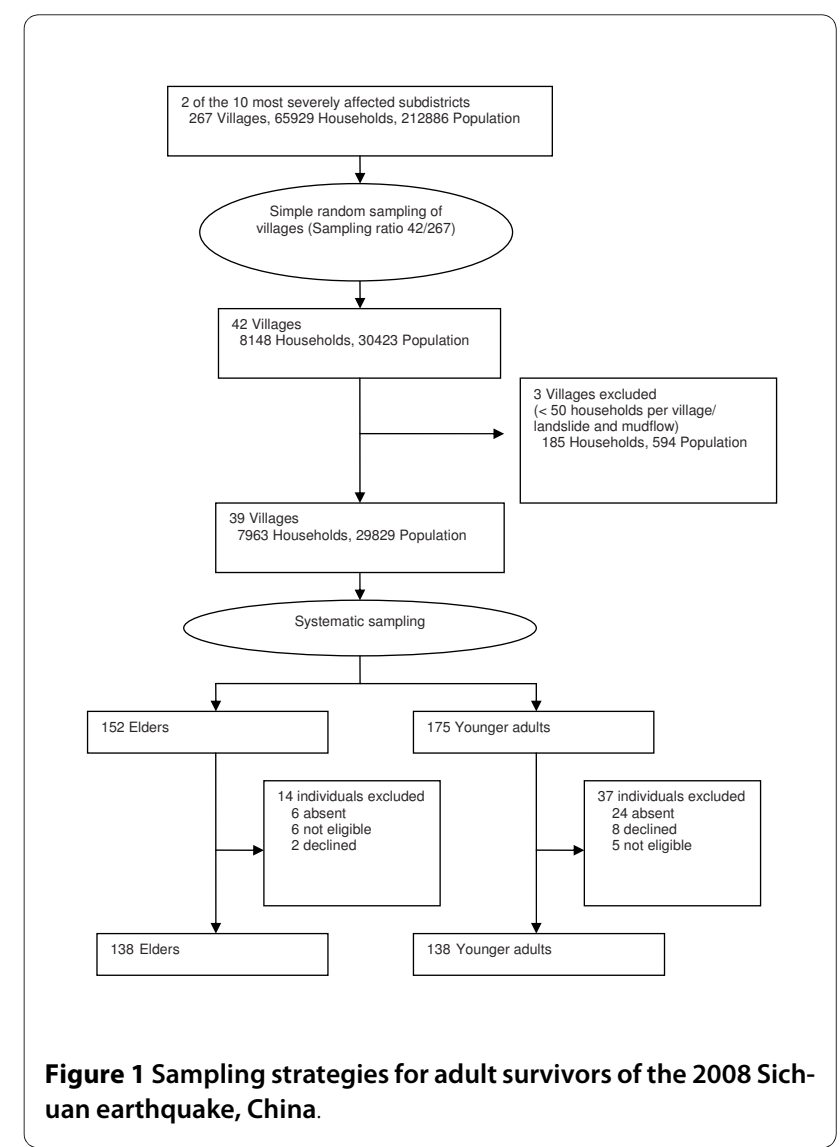


of elders/total number of elders in all of the villages) and then multiplied this ratio by the number of elders in each village [20]. This provided us with the number of elders to be enrolled from each village, proportional to the village size. Next, we calculated a sampling interval (number of elders in the village/number of elders to be sampled from the village), which provided us with the number of people between the two elders to be selected in the sample [20]. At the start of data collection, a randomly generated number between one and ten was used to determine which subject was sampled first; this number was then increased systematically by the sampling interval. Sampling strategies for the younger adults were similar to those of the elderly. As a result, a sample of 152 elders and 175 younger adults was generated, which exceeded the sample size to be required.

\section{Data Collection}

Interviews were carried out by our psychologists (WL, JY, et al.) between August and September, 2009, by which time 15 months had passed since the massive earthquake. A total of 327 adults were targeted. Adults were ineligible if they were not present at the time of the earthquake or had pre-earthquake traumatic experiences (this information was obtained from key informants or the adults themselves, based on which our psychologists judged his or her eligibility). If an adult was not available after two attempts to contact him or her, the adult was omitted and not replaced. A complete description of the survey was presented to the participants before written informed consents were obtained (except the illiterate, who only gave oral consents). The completed questionnaires were carefully examined by the psychologists to ensure that there were no missing values. Adults who were in need of mental health support were referred to local mental health services, and all the visited households were offered a brochure on mental health recovery.

\section{Instruments}

Symptoms of PTSD were measured with the PTSD Checklist-Civilian Version (PCL-C) [21]. The instrument is a self-report 17-item symptom scale that corresponds to DSM-IV criteria [7], and is often used when a clinical interview is not feasible [22,23]. Total score ranges from 17 to 85 , and an adult with a score 50 or greater was classified as having probable PTSD. The Internal consistency of the PCL-C in the present study was 0.88 .

The General Health Questionnaire (GHQ-12) was used to measure the severity of general psychiatric morbidity (i.e., distress and social dysfunction) of the participants $[24,25]$. GHQ-12 scores ranged from 0 to 12 , with higher scores indicating worse conditions; we used a conventional cut-off for the questionnaire (3/4) to define "cases" of general psychiatric morbidity. The Chinese version of the GHQ-12 used in this study has been validated [26,27]. The Internal consistency of the GHQ-12 in the present study was 0.84 .

Earthquake-related experiences were evaluated with an inventory adapted from a prior earthquake exposure scale [28]. Mental health services in the study were defined as interventions conducted by psychologists, psychiatrists, and other certified mental health staff that aimed to promote the mental health recovery of the people surviving the earthquake; people could have received these services in person or in a group, at work or in communities, free or for payment.

The questionnaire was carefully reviewed by our psychologists and linguistic professionals, and was then verified for accuracy and comprehensibility by local mental health staff. The Ethics Committee of the Second Military Medical University approved the procedures and instruments used in the study.

\section{Statistical Analysis}

The PCL-C and GHQ-12 were computed according to the manual. Frequencies, percentages and standard deviations were calculated for descriptive data, $t$-tests were used to evaluate differences in continuous variables, and Chi-square tests were used to test for significance in categorical data. If continuous variables were not normally distributed, Mann-Whitney U tests were applied. Bivariate and backward-conditional multivariate logistic regressions were used to identify independent risk factors for PTSD symptoms and general psychiatric morbidity. All variables with $P<0.05$ in the bivariate analysis were entered into the multivariate models. The entry and removal criteria for the variables in the multivariate analysis were 0.05 and 0.10 , respectively. $P<0.05$ was considered to be statistically significant. Data were analyzed using SPSS version 17.0 (SPSS Inc, Chicago, Ill).

\section{Results}

Information was gathered from 138 elders and 138 younger adults, and the response rates for the two groups were $90.8 \%$ and $78.9 \%$, respectively. Non-response was mostly due to ineligibility or absence. In each group, the respondents and non-respondents did not differ on sex, ethnicity, marital status, or education level. Table 1 shows demographics and earthquake-related experiences of the participants. The Mean (range, SD) age for the elderly and the younger adults was $67.8(60-82,5.9)$ and $33.2(18-$ $59,10.3)$ years $(\mathrm{p}<0.01)$. Compared with the younger adults, the elderly reported higher proportion of being married or living together $(81.2 \%$ vs. $63.8 \%$; $p<0.01)$ and of being primarily or lower-educated (65.2\% vs. $35.5 \%$; $<0.01)$. There were no statistically significant differences between the two groups on the earthquake-related experiences except the loss of family members, as the elders 
Table 1: Participants characteristics - 2008 Sichuan earthquake, China

\begin{tabular}{|c|c|c|c|c|}
\hline \multirow[b]{2}{*}{ Characteristics } & \multicolumn{3}{|c|}{ No. (\%) of participants } & \multirow[b]{2}{*}{ P Value } \\
\hline & $\begin{array}{c}\text { Total } \\
(\mathrm{N}=\mathbf{2 7 6})\end{array}$ & $\begin{array}{l}\text { Elders } \\
(n=138)\end{array}$ & $\begin{array}{l}\text { Younger Adults } \\
\quad(n=138)\end{array}$ & \\
\hline \multicolumn{5}{|l|}{ Demographics } \\
\hline \multicolumn{5}{|l|}{ Sex } \\
\hline Male & $148(53.6)$ & $76(55.1)$ & $72(52.2)$ & 0.63 \\
\hline Female & $128(46.4)$ & $62(44.9)$ & $66(47.8)$ & \\
\hline \multicolumn{5}{|l|}{ Ethnicity } \\
\hline Han & $110(39.9)$ & $54(39.1)$ & $56(40.6)$ & 0.81 \\
\hline Ethnic minorities a & $166(60.1)$ & $84(60.9)$ & $82(59.4)$ & \\
\hline \multicolumn{5}{|l|}{ Marital status } \\
\hline Married or living together & $200(72.5)$ & $112(81.2)$ & $88(63.8)$ & 0.001 \\
\hline Others (unmarried, divorced, etc.) & $76(27.5)$ & $26(18.8)$ & $50(36.2)$ & \\
\hline \multicolumn{5}{|l|}{ Education level } \\
\hline Primary school or lower & $139(50.4)$ & $90(65.2)$ & $49(35.5)$ & $<0.001$ \\
\hline Higher than primary school & $137(49.6)$ & $48(34.8)$ & $89(64.5)$ & \\
\hline \multicolumn{5}{|l|}{ Earthquake-related experiences } \\
\hline \multicolumn{5}{|l|}{ Having been in serious danger } \\
\hline Yes & $167(60.5)$ & $84(60.9)$ & $83(60.1)$ & 0.90 \\
\hline No & $109(39.5)$ & $54(39.1)$ & $55(39.9)$ & \\
\hline \multicolumn{5}{|l|}{ Having been seriously injured } \\
\hline Yes & $33(12.0)$ & $19(13.8)$ & $14(10.1)$ & 0.35 \\
\hline No & $243(88.0)$ & $119(86.2)$ & $124(89.9)$ & \\
\hline \multicolumn{5}{|l|}{$\begin{array}{l}\text { Having family members or friends } \\
\text { seriously injured }\end{array}$} \\
\hline Yes & $49(17.8)$ & $26(18.8)$ & $23(16.7)$ & 0.64 \\
\hline No & $227(82.2)$ & $112(81.2)$ & $115(83.3)$ & \\
\hline \multicolumn{5}{|l|}{$\begin{array}{l}\text { Having witnessed someone being } \\
\text { killed or seriously injured }\end{array}$} \\
\hline Yes & $140(50.7)$ & $70(50.7)$ & $70(50.7)$ & 1.00 \\
\hline No & $136(49.3)$ & $68(49.3)$ & $68(49.3)$ & \\
\hline \multicolumn{5}{|l|}{ Having lost family members } \\
\hline Yes & $38(13.8)$ & $26(18.8)$ & $12(8.7)$ & 0.01 \\
\hline No & $238(86.2)$ & $112(81.2)$ & $126(91.3)$ & \\
\hline \multicolumn{5}{|l|}{ Having lost significant others } \\
\hline Yes & $100(36.2)$ & $49(35.5)$ & $51(37.0)$ & 0.80 \\
\hline No & $176(63.8)$ & $89(64.5)$ & $87(63.0)$ & \\
\hline \multicolumn{5}{|l|}{$\begin{array}{l}\text { Having one's house seriously } \\
\text { damaged }\end{array}$} \\
\hline Yes & $227(82.2)$ & $117(84.8)$ & $110(79.7)$ & 0.27 \\
\hline No & $49(17.8)$ & $21(15.2)$ & $28(20.3)$ & \\
\hline \multicolumn{5}{|l|}{ Having lost important belongings $b$} \\
\hline Yes & $113(40.9)$ & $61(44.2)$ & $52(37.7)$ & 0.27 \\
\hline No & $163(59.1)$ & $77(55.8)$ & $86(62.3)$ & \\
\hline
\end{tabular}


Table 1: Participants characteristics - 2008 Sichuan earthquake, China (Continued)

\begin{tabular}{|c|c|c|c|c|}
\hline \multicolumn{5}{|c|}{$\begin{array}{l}\text { Having felt extremely anxious about } \\
\text { one's own life }\end{array}$} \\
\hline Yes & $226(81.9)$ & $112(81.2)$ & $114(82.6)$ & 0.76 \\
\hline No & $50(18.1)$ & $26(18.8)$ & 24 (17.4) & \\
\hline \multicolumn{5}{|c|}{$\begin{array}{l}\text { Having felt scared that family } \\
\text { members or significant others would } \\
\text { die or be seriously injured }\end{array}$} \\
\hline Yes & $253(91.7)$ & $128(92.8)$ & $125(90.6)$ & 0.51 \\
\hline No & $23(8.3)$ & $10(7.2)$ & $13(9.4)$ & \\
\hline \multicolumn{5}{|c|}{$\begin{array}{l}\text { Having felt guilt concerning } \\
\text { someone's death or injury }\end{array}$} \\
\hline Yes & $121(43.8)$ & $58(42.0)$ & $63(45.7)$ & 0.54 \\
\hline No & $155(56.2)$ & $80(58.0)$ & $75(54.3)$ & \\
\hline
\end{tabular}

a Ethnic minorities refer to non-Han population in China, which account for a larger population in the survey areas than Han people.

${ }^{b}$ Important belongings refer to physical possessions of sentimental value to the participants.

were more likely to report the loss of family members than the younger adults $(18.8 \%$ vs. $8.7 \%$; $\mathrm{p}=0.01)$.

The point prevalence rates of PTSD symptoms among the elderly and younger adults were $22.5 \%$ and $8.0 \%(\mathrm{p}=$ 0.001 ), respectively. The point prevalence rates of general psychiatric morbidity among the elderly and younger adults were $42.0 \%$ and $25.4 \%(\mathrm{p}=0.003)$, respectively. The younger adults reported more utilization of mental health services $(19.6 \%$ vs. $12.3 \%)$, but the difference is not significant $(\mathrm{p}=0.10)$.

Table 2 shows the risk factors for PTSD symptoms among the adult survivors. In the bivariate analysis, a significantly higher prevalence of PTSD symptoms were found among adults who were elderly, reported having been in serious danger, having been seriously injured, having lost family members, having lost significant others, having felt guilt concerning one's death or injury, or having not utilized mental health services. In the multivariate analysis, being elderly (odds ratio $(\mathrm{OR})=3.56$, $95 \%$ confidence interval $(\mathrm{CI}): 1.57,8.06 ; \mathrm{p}=0.002)$, having been in serious danger $(\mathrm{OR}=3.12,95 \% \mathrm{CI}$ : 1.32, 7.37; $\mathrm{p}=0.009)$, having lost family members $(\mathrm{OR}=3.43,95 \%$ CI: 1.46, 8.02; $\mathrm{p}=0.005)$, and having felt guilt concerning one's death or injury ( $\mathrm{OR}=5.22,95 \% \mathrm{CI}: 2.32,11.75$; $\mathrm{p}<$ 0.001 ) were significantly and independently associated with PTSD symptoms.

Table 3 shows the risk factors for symptoms of general psychiatric morbidity among the adult survivors. In the bivariate analysis, being elderly, having been seriously injured, having family members or friends seriously injured, having witnessed someone being killed or seriously injured, having lost family members, having lost significant others, having lost important belongings, having felt guilt concerning one's death or injury, and having not utilized mental health services were significantly associated with general psychiatric morbidity. In the multivariate analysis, being elderly $(\mathrm{OR}=2.14,95 \% \mathrm{CI}: 1.25$, $3.66 ; \mathrm{p}=0.005$ ), having family members or friends seriously injured (OR $=2.13,95 \% \mathrm{CI}: 1.10,4.11 ; \mathrm{p}=0.03$ ), and having felt guilt concerning one's death or injury $(\mathrm{OR}=$ 2.0, 95\% CI: $1.18,3.41 ; \mathrm{p}=0.01$ ) were significantly and independently associated with general psychiatric morbidity. It is noteworthy that although the utilization of mental health services was not significantly associated with PTSD or general psychiatric morbidity in the multivariate analysis, it was still one of the independent risk factors for both of the symptoms.

\section{Discussion}

In the study, significant distinctions were found in the prevalence of PTSD $(22.5 \%$ vs. $8.0 \%)$ and general psychiatric morbidity $(42.0 \%$ vs. $25.4 \%)$ between elder and younger adult survivors. Arguments may exist on whether the outcome can truly reflect the psychological consequences of the disaster, as it might have been confounded by pre-existing conditions. However, studies show that the PTSD prevalence in the general population of China is well below $1 \%$, and no age difference is reported $[29,30]$. This adds much confidence to our conclusion that the elderly were more psychologically vulnerable to natural disasters. Nevertheless, the conclusion maybe biased by the limited sample size, case detection technique, and the cross-sectional nature of the study; the imbalanced physical conditions, traumatic exposures, and utilization of mental health services between elder and younger adult survivors may also play a role in interpreting the conclusion. But even after the outcomes were adjusted by a number of confounders using stringent bivariate and multivariate analyses, the post-disaster vul- 
Table 2: Bivariate and multivariate analysis of PTSD symptoms among the adult survivors

\begin{tabular}{|c|c|c|c|c|c|}
\hline & $\begin{array}{l}\text { PTSD, } \\
\text { No. (\%) }\end{array}$ & $\begin{array}{c}\text { Bivariate OR } \\
(95 \% \mathrm{Cl})\end{array}$ & P Value & $\begin{array}{c}\text { Multivariate } \\
\text { OR } \\
(95 \% \mathrm{Cl})\end{array}$ & P Value \\
\hline Overall & $42(15.2)$ & & & & \\
\hline \multicolumn{6}{|l|}{ Demographics } \\
\hline \multicolumn{6}{|l|}{ Age group } \\
\hline $\begin{array}{l}\text { Younger adults }(<60 \mathrm{y}) \\
\text { Elders }(\geq 60 \mathrm{y})\end{array}$ & $\begin{array}{c}11(8.0) \\
31(22.5)\end{array}$ & $\begin{array}{c}1.00 \\
3.35(1.61- \\
6.97)\end{array}$ & 0.001 & $\begin{array}{c}1.00 \\
3.56(1.57- \\
8.06)\end{array}$ & 0.002 \\
\hline \multicolumn{6}{|l|}{ Sex } \\
\hline $\begin{array}{l}\text { Male } \\
\text { Female }\end{array}$ & $\begin{array}{l}17(11.5) \\
25(19.5)\end{array}$ & $\begin{array}{c}1.00 \\
1.87(0.96- \\
3.65)\end{array}$ & 0.07 & & \\
\hline \multicolumn{6}{|l|}{ Ethnicity } \\
\hline Han & $17(15.5)$ & $\begin{array}{l}1.03(0.53- \\
2.01)\end{array}$ & & & \\
\hline Ethnic minorities & $25(15.1)$ & 1.00 & 0.93 & & \\
\hline \multicolumn{6}{|l|}{ Marital status } \\
\hline $\begin{array}{l}\text { Married or living together } \\
\text { Others (unmarried, } \\
\text { divorced, etc.) }\end{array}$ & $\begin{array}{l}32(16.0) \\
10(13.2)\end{array}$ & $\begin{array}{c}1.26(0.59- \\
2.70) \\
1.00\end{array}$ & 0.56 & & \\
\hline \multicolumn{6}{|l|}{ Education level } \\
\hline $\begin{array}{l}\text { Primary school or lower } \\
\text { Higher than primary } \\
\text { school }\end{array}$ & $\begin{array}{l}26(18.7) \\
16(11.7)\end{array}$ & $\begin{array}{c}1.74(0.89- \\
3.41) \\
1.00\end{array}$ & 0.11 & & \\
\hline \multicolumn{6}{|l|}{ Earthquake-related experiences } \\
\hline \multicolumn{6}{|l|}{ Having been in serious danger } \\
\hline $\begin{array}{l}\text { Yes } \\
\text { No }\end{array}$ & $\begin{array}{c}33(19.8) \\
9(8.3)\end{array}$ & $\begin{array}{l}2.74(1.25- \\
5.98) \\
1.00\end{array}$ & 0.01 & $\begin{array}{c}3.12(1.32- \\
7.37) \\
1.00\end{array}$ & 0.009 \\
\hline \multicolumn{6}{|l|}{ Having been seriously injured } \\
\hline $\begin{array}{l}\text { Yes } \\
\text { No }\end{array}$ & $\begin{array}{l}10(30.3) \\
32(13.2)\end{array}$ & $\begin{array}{l}2.87(1.25- \\
6.58) \\
1.00\end{array}$ & 0.01 & NA & \\
\hline \multicolumn{6}{|l|}{$\begin{array}{l}\text { Having family members or } \\
\text { friends seriously injured }\end{array}$} \\
\hline $\begin{array}{l}\text { Yes } \\
\text { No }\end{array}$ & $\begin{array}{l}11(22.4) \\
31(13.7)\end{array}$ & $\begin{array}{c}1.83(0.85- \\
3.96) \\
1.00\end{array}$ & 0.12 & & \\
\hline \multicolumn{6}{|l|}{$\begin{array}{l}\text { Having witnessed someone } \\
\text { being killed or seriously injured }\end{array}$} \\
\hline Yes & $27(19.3)$ & $\begin{array}{l}1.93(0.98- \\
3.81)\end{array}$ & & & \\
\hline $\begin{array}{l}\text { Yes } \\
\text { No }\end{array}$ & $\begin{array}{l}27(19.3) \\
15(11.0)\end{array}$ & $\begin{array}{c}1.93(0.98- \\
3.81) \\
1.00\end{array}$ & 0.06 & & \\
\hline \multicolumn{6}{|l|}{ Having Lost family members } \\
\hline $\begin{array}{l}\text { Yes } \\
\text { No }\end{array}$ & $\begin{array}{l}15(39.5) \\
27(11.3)\end{array}$ & $\begin{array}{c}5.10(2.37- \\
10.94) \\
1.00\end{array}$ & $<0.001$ & $\begin{array}{l}3.43(1.46- \\
8.02) \\
1.00\end{array}$ & 0.005 \\
\hline
\end{tabular}


Table 2: Bivariate and multivariate analysis of PTSD symptoms among the adult survivors (Continued)

\begin{tabular}{|c|c|c|c|c|c|}
\hline \multicolumn{6}{|c|}{ Having Lost significant others } \\
\hline $\begin{array}{l}\text { Yes } \\
\text { No }\end{array}$ & $\begin{array}{l}23(23.0) \\
19(10.8)\end{array}$ & $\begin{array}{l}2.47(1.27- \\
4.80) \\
1.00\end{array}$ & 0.008 & NA & \\
\hline \multicolumn{6}{|c|}{$\begin{array}{l}\text { Having one's house seriously } \\
\text { damaged }\end{array}$} \\
\hline $\begin{array}{l}\text { Yes } \\
1.00\end{array}$ & $\begin{array}{c}36(15.9) \\
6(12.2)\end{array}$ & $\begin{array}{l}1.35(0.54- \\
3.41) \\
1.00\end{array}$ & 0.52 & & \\
\hline \multicolumn{6}{|c|}{$\begin{array}{l}\text { Having Lost important } \\
\text { belongings }\end{array}$} \\
\hline $\begin{array}{l}\text { Yes } \\
\text { No }\end{array}$ & $\begin{array}{l}21(18.6) \\
21(12.9)\end{array}$ & $\begin{array}{l}1.54(0.80- \\
2.98) \\
1.00\end{array}$ & 0.20 & & \\
\hline \multicolumn{6}{|c|}{$\begin{array}{l}\text { Having felt extremely anxious } \\
\text { about one's own life }\end{array}$} \\
\hline $\begin{array}{l}\text { Yes } \\
\text { No }\end{array}$ & $\begin{array}{l}34(15.0) \\
8(16.0)\end{array}$ & $\begin{array}{l}1.00 \\
1.08(0.47- \\
2.49)\end{array}$ & 0.87 & & \\
\hline \multicolumn{6}{|c|}{$\begin{array}{l}\text { Having felt scared that family } \\
\text { members or significant } \\
\text { others would die or be } \\
\text { seriously injured }\end{array}$} \\
\hline $\begin{array}{l}\text { Yes } \\
\text { No }\end{array}$ & $\begin{array}{l}40(15.8) \\
2(8.7)\end{array}$ & $\begin{array}{l}1.97(0.45- \\
8.74) \\
1.00\end{array}$ & 0.37 & & \\
\hline \multicolumn{6}{|c|}{$\begin{array}{l}\text { Having felt guilt concerning } \\
\text { someone's death or injury }\end{array}$} \\
\hline $\begin{array}{l}\text { Yes } \\
\text { No }\end{array}$ & $\begin{array}{l}31(25.6) \\
11(7.1)\end{array}$ & $\begin{array}{l}4.51(2.16- \\
9.42) \\
1.00\end{array}$ & $<0.001$ & $\begin{array}{c}5.22(2.32- \\
11.75) \\
1.00\end{array}$ & $<0.001$ \\
\hline \multicolumn{6}{|c|}{$\begin{array}{l}\text { Utilization of mental health } \\
\text { services }\end{array}$} \\
\hline $\begin{array}{l}\text { Yes } \\
\text { No }\end{array}$ & $\begin{array}{c}1(2.3) \\
41(17.7)\end{array}$ & $\begin{array}{c}1.00 \\
9.23(1.24- \\
68.97)\end{array}$ & 0.03 & $\begin{array}{l}1.00 \\
7.31(0.93- \\
57.23)\end{array}$ & 0.06 \\
\hline
\end{tabular}

Notes: NA (not available), is a marker for variables dropped out of the multivariate model.

nerability to PTSD and general psychiatric morbidity is still age-dependent.

Studies show that natural disasters may result in feelings of fear, helplessness and vulnerability in many people of all ages [31,32]. The underlying mechanisms that the elderly were more likely to develop psychological problems after disasters are still unclear. But there are some potential reasons. Pekovic et al. argued that due to the fact that an elder often already feels frail because of chronic health conditions, impaired cognitive abilities and decreased sensory awareness, the impact of an unexpected disaster may be overwhelming [3]. Taylor and Vidovic et al. discussed the effect of adrenergic system and the hypothalamic-pituitary-adrenal axis on the neurobiology of PTSD $[33,34]$, although there is no current evi- dence that the changes seen in these systems with aging affect the development or presentation of post-disaster PTSD in older individuals. Further basic researches are needed to focus on the mechanisms why post-disaster vulnerability to psychological problems is likely to be agedependent, and provide sound proofs for interventions.

Despite the fact that the work of mental health recovery was critical following the disaster, the road for mental health recovery seems uncertain. Davidson et al. reported that PTSD became chronic in $46 \%$ of all patients who developed the disorder [35], and Kessler et al. found that one-third of individuals who developed PTSD after trauma did not have remission of the disorder after ten years [36]. Moreover, past experience in Japan, the Pakistan earthquake, and Hurricane Katrina suggests that 
Table 3: Bivariate and multivariate analysis of general psychiatric morbidity among the adult survivors

\begin{tabular}{|c|c|c|c|c|c|}
\hline & $\begin{array}{r}\text { General } \\
\text { Psychiatric } \\
\text { Morbidity } \\
\text { No. (\%) }\end{array}$ & $\begin{array}{c}\text { Bivariate OR } \\
(95 \% \mathrm{CI})\end{array}$ & P Value & $\begin{array}{c}\text { Multivariate } \\
\text { OR } \\
(95 \% \mathrm{CI})\end{array}$ & P Value \\
\hline Overall & $93(33.7)$ & & & & \\
\hline \multicolumn{6}{|l|}{ Demographics } \\
\hline \multicolumn{6}{|l|}{ Age group } \\
\hline $\begin{array}{l}\text { Younger adults }(<60 \mathrm{y}) \\
\text { Elders }(\geq 60 \mathrm{y})\end{array}$ & $\begin{array}{l}35(25.4) \\
58(42.0)\end{array}$ & $\begin{array}{l}1.00 \\
2.13(1.28- \\
3.56)\end{array}$ & 0.004 & $\begin{array}{l}1.00 \\
2.14(1.25- \\
\quad 3.66)\end{array}$ & 0.005 \\
\hline \multicolumn{6}{|l|}{ Sex } \\
\hline $\begin{array}{l}\text { Male } \\
\text { Female }\end{array}$ & $\begin{array}{l}43(29.1) \\
50(39.1)\end{array}$ & $\begin{array}{l}1.00 \\
1.57(0.95- \\
2.59)\end{array}$ & 0.08 & & \\
\hline \multicolumn{6}{|l|}{ Ethnicity } \\
\hline $\begin{array}{l}\text { Han } \\
\text { Ethnic minorities }\end{array}$ & $\begin{array}{l}38(34.5) \\
55(33.1)\end{array}$ & $\begin{array}{c}1.07(0.64- \\
1.77) \\
1.00\end{array}$ & 0.81 & & \\
\hline \multicolumn{6}{|l|}{ Marital status } \\
\hline $\begin{array}{l}\text { Married or living together } \\
\text { Others (unmarried, } \\
\text { divorced, etc.) }\end{array}$ & $\begin{array}{l}72(36.0) \\
21(27.6)\end{array}$ & $\begin{array}{c}1.47(0.83- \\
2.63) \\
1.00\end{array}$ & 0.19 & & \\
\hline \multicolumn{6}{|l|}{ Education level } \\
\hline $\begin{array}{l}\text { Primary school or lower } \\
\text { Higher than primary } \\
\text { school }\end{array}$ & $\begin{array}{l}46(33.1) \\
47(34.3)\end{array}$ & $\begin{array}{c}1.00 \\
1.06(0.64- \\
1.74)\end{array}$ & 0.83 & & \\
\hline \multicolumn{6}{|l|}{ Earthquake-related experiences } \\
\hline \multicolumn{6}{|l|}{ Having been in serious danger } \\
\hline $\begin{array}{l}\text { Yes } \\
\text { No }\end{array}$ & $\begin{array}{l}63(37.7) \\
30(27.5)\end{array}$ & $\begin{array}{c}1.60(0.95- \\
2.69) \\
1.00\end{array}$ & 0.08 & & \\
\hline \multicolumn{6}{|l|}{ Having been seriously injured } \\
\hline $\begin{array}{l}\text { Yes } \\
\text { No }\end{array}$ & $\begin{array}{l}17(51.5) \\
76(31.3)\end{array}$ & $\begin{array}{c}2.34(1.12- \\
4.87) \\
1.00\end{array}$ & 0.02 & NA & \\
\hline \multicolumn{6}{|l|}{$\begin{array}{l}\text { Having family members or } \\
\text { friends seriously injured }\end{array}$} \\
\hline $\begin{array}{l}\text { Yes } \\
\text { No }\end{array}$ & $\begin{array}{l}25(51.0) \\
68(30.0)\end{array}$ & $\begin{array}{c}2.44(1.30- \\
4.56) \\
1.00\end{array}$ & 0.005 & $\begin{array}{c}2.13(1.10- \\
4.11) \\
1.00\end{array}$ & 0.03 \\
\hline \multicolumn{6}{|l|}{$\begin{array}{l}\text { Having witnessed someone } \\
\text { being killed or seriously injured }\end{array}$} \\
\hline $\begin{array}{l}\text { Yes } \\
\text { No }\end{array}$ & $\begin{array}{l}57(40.7) \\
36(26.5)\end{array}$ & $\begin{array}{l}1.91(1.15- \\
3.17) \\
1.00\end{array}$ & 0.01 & NA & \\
\hline \multicolumn{6}{|l|}{ Having Lost family members } \\
\hline $\begin{array}{l}\text { Yes } \\
\text { No }\end{array}$ & $\begin{array}{l}21(55.3) \\
72(30.3)\end{array}$ & $\begin{array}{l}2.85(1.42- \\
5.72) \\
1.00\end{array}$ & 0.003 & NA & \\
\hline
\end{tabular}

Having Lost significant others 
Table 3: Bivariate and multivariate analysis of general psychiatric morbidity among the adult survivors (Continued)

\begin{tabular}{|c|c|c|c|c|c|}
\hline $\begin{array}{l}\text { Yes } \\
\text { No }\end{array}$ & $\begin{array}{l}41(41.0) \\
52(29.5)\end{array}$ & $\begin{array}{c}1.66(0.99- \\
2.77) \\
1.00\end{array}$ & 0.05 & $\mathrm{NA}$ & \\
\hline \multicolumn{6}{|c|}{$\begin{array}{l}\text { Having one's house seriously } \\
\text { damaged }\end{array}$} \\
\hline $\begin{array}{l}\text { Yes } \\
\text { No }\end{array}$ & $\begin{array}{l}81(35.7) \\
12(24.5)\end{array}$ & $\begin{array}{l}1.71(0.85- \\
3.46) \\
1.00\end{array}$ & 0.14 & & \\
\hline \multicolumn{6}{|c|}{$\begin{array}{l}\text { Having Lost important } \\
\text { belongings }\end{array}$} \\
\hline $\begin{array}{l}\text { Yes } \\
\text { No }\end{array}$ & $\begin{array}{l}47(41.6) \\
46(28.2)\end{array}$ & $\begin{array}{l}1.81(1.09- \\
3.01) \\
1.00\end{array}$ & 0.02 & $\begin{array}{c}1.60(0.94- \\
2.72) \\
1.00\end{array}$ & 0.09 \\
\hline \multicolumn{6}{|c|}{$\begin{array}{l}\text { Having felt extremely anxious } \\
\text { about one's own life }\end{array}$} \\
\hline $\begin{array}{l}\text { Yes } \\
\text { No }\end{array}$ & $\begin{array}{l}78(34.5) \\
15(30.0)\end{array}$ & $\begin{array}{c}1.23(0.63- \\
2.39) \\
1.00\end{array}$ & 0.54 & & \\
\hline \multicolumn{6}{|c|}{$\begin{array}{l}\text { Having felt scared that family } \\
\text { members or significant } \\
\text { others would die or be } \\
\text { seriously injured }\end{array}$} \\
\hline $\begin{array}{l}\text { Yes } \\
\text { No }\end{array}$ & $\begin{array}{c}89(35.2) \\
4(17.4)\end{array}$ & $\begin{array}{l}2.58(0.85- \\
7.81) \\
1.00\end{array}$ & 0.09 & & \\
\hline \multicolumn{6}{|c|}{$\begin{array}{l}\text { Having felt guilt concerning } \\
\text { someone's death or injury }\end{array}$} \\
\hline $\begin{array}{l}\text { Yes } \\
\text { No }\end{array}$ & $\begin{array}{l}52(43.0) \\
41(26.5)\end{array}$ & $\begin{array}{l}2.10(1.26- \\
3.48) \\
1.00\end{array}$ & 0.004 & $\begin{array}{l}2.00(1.18- \\
3.41) \\
1.00\end{array}$ & 0.01 \\
\hline \multicolumn{6}{|c|}{$\begin{array}{l}\text { Utilization of mental health } \\
\text { services }\end{array}$} \\
\hline $\begin{array}{l}\text { Yes } \\
\text { No }\end{array}$ & $\begin{array}{c}9(20.5) \\
84(36.2)\end{array}$ & $\begin{array}{c}1.00 \\
.21(1.01-4.82)\end{array}$ & 0.05 & $\begin{array}{c}1.00 \\
2.05(0.91- \\
4.63)\end{array}$ & 0.08 \\
\hline
\end{tabular}

Notes: NA(not available) is a marker for variables dropped out of the multivariate model.

chronic medical needs post-disaster are often inadequately managed and can result in increased rates of complications and indirect morbidity after a disaster [3739 ]. In our study, we found that only $2.4 \%$ of the survivors with PTSD and $9.7 \%$ of the survivors with general psychiatric morbidity reported utilization of mental health services, which suggested that a far greater proportion of people with mental health problems had not accessed appropriate care yet. It is also noteworthy that, despite the more crucial situation of psychopathology that elder survivors met, they utilized less mental health services. Additionally, when the elderly were involved in the impaired psychological recovery, the disaster-related impact seems more severe and lasting than we thought [40].
The findings of our study may provide a better understanding of survivors' mental health status after the earthquake. Being elderly, having been in serious danger, having lost family members, having felt guilt concerning one's death or injury, and having not utilized mental health services were independent risk factors for PTSD symptoms, while being elderly, having family members or friends seriously injured, having lost important belongings, having felt guilt concerning one's death or injury, and having not utilized mental health services were independent risk factors for general psychiatric morbidity. These results may help to identify the survivors with an increased risk for either PTSD or psychiatric morbidity. As disaster-related psychological sequelae may last for many years $[41,42]$, actions should be taken to minimize the possible negative impacts. A comprehensive screen- 
ing program should be established to evaluate the mental health conditions of survivors in earthquake-affected areas at regular intervals, in order to facilitate the early identification of people with mental health problems. Previous studies have also demonstrated the value of community-based mental health aid [43,44]. As the survivors tend not to come for help, outreach services in the community may be effective. It is noteworthy that, the mental health response should not be segregated from the other interventions, because the people not only have psychological needs but also require physical, economic, and spiritual supports as well.

Certain limitations of the study should be recognized. Sichuan earthquake was so powerful and extensive that the shock spread almost half of mainland China. Places had different casualties and losses, and it's hard to select a bunch of people without experiencing the disaster; or when the people were identified as non-exposed, sociodemographics and cultures varied greatly from the exposed. Therefore, it's almost impossible for us to provide comparisons between exposed and non-exposed group within the elder and younger adult survivors. However, we retrieved data from the general population to examine the effect of pre-existing conditions, which added confidence to our conclusions.

\section{Conclusion}

Compared with the younger adults, the elderly survivors were more likely to develop PTSD and general psychiatric morbidity. Despite utilization of mental health services may markedly improve the mental health status, it has been only provided to less than ten percent of the survivors with the mental health symptoms. More mental health services should be distributed to the elderly and groups at particular risk, to ensure their smooth mental health reconstruction after the earthquake.

\section{Competing interests}

The authors declare that they have no competing interests.

\section{Authors' contributions}

ZJ and WT made substantial contributions to conception, design, and interpretation of the data and they involved in drafting the manuscript. All authors participated in the design and performing of the study, acquisition of data and helped to draft the manuscript or revising it. YC performed the statistical analysis. All authors read and approved the final manuscript.

\section{Acknowledgements}

This study was supported by Shanghai Leading Academic Discipline Project, Project Number: B907, and Disaster Research Fund of Second Military Medical University.

\section{Author Details}

'Faculty of Health Service, Second Military Medical University, Shanghai, China, 2Department of Psychology, Second Military Medical University, Shanghai, China, ${ }^{3}$ Department of Health Statistics, Second Military Medical University, Shanghai, China and ${ }^{4}$ Centers for Disease Control and Prevention, Aba Tibetan and Qiang Autonomous Prefecture, Sichuan province, China
Received: 29 December 2009 Accepted: 30 March 2010

Published: 30 March 2010

\section{References}

1. Chan EY: The untold stories of the Sichuan earthquake. Lancet 2008 , 372:359-362.

2. Centers for Disease Control and Prevention: Rapid Assessment of the Needs and Health Status of Older Adults After Hurricane Charley -Charlotte, DeSoto, and Hardee Counties, Florida, August 27--31, 2004. [http://www.cdc.gov/mmwr/preview/mmwrhtml/mm5336a2.htm].

3. Pekovic $V$, Seff $L$, Rothman MB: Planning for and responding to special needs of elders in natural disasters. Generations 2007, 31:37-41.

4. Goenjian AK, Najarian LM, Pynoos RS, Steinberg AM, Manoukian G, Tavosian A, Fairbanks LA: Posttraumatic stress disorder in elderly and younger adults after the 1988 earthquake in Armenia. Am J Psychiatry 1994, 151:895-901.

5. van Griensven F, Chakkraband ML, Thienkrua W, Pengjuntr W, Lopes Cardozo B, Tantipiwatanaskul P, Mock PA, Ekassawin S, Varangrat A,

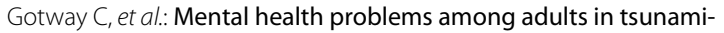
affected areas in southern Thailand. JAMA 2006, 296:537-548.

6. Wang X, Gao L, Shinfuku N, Zhang H, Zhao C, Shen Y: Longitudinal study of earthquake-related PTSD in a randomly selected community sample in north China. Am J Psychiatry 2000, 157:1260-1266.

7. American Psychiatric Association: Diagnostic and Statistical Manual of Mental Disorders DSM-IV-TR Fourth edition. Washington, DC: American Psychiatric Association; 2000.

8. Neria Y, Nandi A, Galea S: Post-traumatic stress disorder following disasters: a systematic review. Psychol Med 2008, 38:467-480.

9. Liu A, Tan H, Zhou J, Li S, Yang T, Wang J, Liu J, Tang X, Sun Z, Wen SW: An epidemiologic study of posttraumatic stress disorder in flood victims in Hunan China. Can J Psychiatry 2006, 51:350-354.

10. Ticehurst S, Webster RA, Carr VJ, Lewin TJ: The psychosocial Impact of an earthquake on the elderly. International Journal of Geriatric Psychiatry 1999, 11:943-951.

11. Cherniack EP: The impact of natural disasters on the elderly. Am J Disaster Med 2008, 3:133-139.

12. Kohn R, Levav I, Garcia ID, Machuca ME, Tamashiro R: Prevalence, risk factors and aging vulnerability for psychopathology following a natural disaster in a developing country. Int J Geriatr Psychiatry 2005 20:835-841.

13. State Council Information Office of the People's Republic of China: Latest Developments of Wenchuan Earthquake Relief by September 25, 2008. 2008 [http://www.scio.gov.cn/zxbd/wz/200905/t310218.htm].

14. State Council Information Office of the People's Republic of China: 845.1 billion yuan were lost in Sichuan earthquake. 2008 [http:// www.china.com.cn/news/2008-09/04/content 16386369.htm].

15. Lopes Cardozo B, Talley L, Burton A, Crawford C: Karenni refugees living in Thai-Burmese border camps: traumatic experiences, mental health outcomes, and social functioning. Soc Sci Med 2004, 58:2637-2644.

16. Feng S, Tan H, Benjamin A, Wen S, Liu A, Zhou J, Li S, Yang T, Zhang Y, Li X, $\mathrm{Li}$ G: Social support and posttraumatic stress disorder among flood victims in Hunan, China. Ann Epidemiol 2007, 17:827-833.

17. Chen CH, Tan HK, Liao LR, Chen HH, Chan CC, Cheng JJ, Chen CY, Wang TN, Lu ML: Long-term psychological outcome of 1999 Taiwan earthquake survivors: a survey of a high-risk sample with property damage. Compr Psychiatry 2007, 48:269-275.

18. Wan GH: Peasant flood in China: internal migration and its policy determinants. Third World Q 1995, 16:173-196.

19. Ministry of Civil Affaires: Ten most severely affected subdistricts are defined in Sichuan province. 2008 [http://www.gov.cn/jrzg/2008-07/ 22/content 1053017.htm

20. Rubin A, Babbie ER: Research Methods for Social Work 6th edition. Beijing: Peking University Press; 2008.

21. Blanchard EB, Jones-Alexander J, Buckley TC, Forneris CA: Psychometric properties of the PTSD Checklist (PCL). Behav Res Ther 1996, 34:669-673.

22. Dobie DJ, Kivlahan DR, Maynard C, Bush KR, McFall M, Epler AJ, Bradley KA: Screening for post-traumatic stress disorder in female Veteran's Affairs patients: validation of the PTSD checklist. Gen Hosp Psychiatry 2002, 24:367-374 
23. Terhakopian A, Sinaii N, Engel CC, Schnurr PP, Hoge CW: Estimating population prevalence of posttraumatic stress disorder: an example using the PTSD checklist. J Trauma Stress 2008, 21:290-300.

24. Goldberg D, Williams P: A user's guide to the general health questionnaire Windsor: NFER-Nelson; 1988

25. Goldberg DP, Gater R, Sartorius N, Ustun TB, Piccinelli M, Gureje O, Rutter $\mathrm{C}$ : The validity of two versions of the GHQ in the WHO study of menta illness in general health care. Psychol Med 1997, 27:191-197.

26. Chan DW, Chan TS: Reliability, validity and the structure of the General Health Questionnaire in a Chinese context. Psychol Med 1983, 13:363-371

27. Pan PC, Goldberg DP: A comparison of the validity of GHQ-12 and CHQ12 in Chinese primary care patients in Manchester. Psychol Med 1990, 20:931-940.

28. Roussos A, Goenjian AK, Steinberg AM, Sotiropoulou C, Kakaki M, Kabakos C, Karagianni S, Manouras V: Posttraumatic stress and depressive reactions among children and adolescents after the 1999 earthquake in Ano Liosia, Greece. Am J Psychiatry 2005, 162:530-537.

29. Phillips MR, Zhang J, Shi Q, Song Z, Ding Z, Pang S, Li X, Zhang Y, Wang Z: Prevalence, treatment, and associated disability of mental disorders in four provinces in China during 2001 - 05: an epidemiological survey. Lancet 2009, 373:2041-2053.

30. LU B, Wang C, Chen H, Zhang C, Liu M: Comparisons between different districts on the prevalence of posttraumatic stress disorder. Journal of Psychiatry 2009, 22:373-374.

31. Hibino Y, Takaki J, Kambayashi Y, Hitomi Y, Sakai A, Sekizuka N, Ogino K, Nakamura H: Health impact of disaster-related stress on pregnant women living in the affected area of the Noto Peninsula earthquake in Japan. Psychiatry Clin Neurosci 2009, 63:107-115.

32. Becker SM: Psychosocial care for adult and child survivors of the tsunami disaster in India. J Child Adolesc Psychiatr Nurs 2007, 20:148-155.

33. Taylor F, Raskind MA: The alpha1-adrenergic antagonist prazosin improves sleep and nightmares in civilian trauma posttraumatic stress disorder. J Clin Psychopharmacol 2002, 22:82-85.

34. Vidovic A, Vilibic M, Sabioncello A, Gotovac K, Rabatic S, Folnegovic-Smalc $\checkmark$, Dekaris D: Circulating lymphocyte subsets, natural killer cell cytotoxicity, and components of hypothalamic-pituitary-adrenal axis in Croatian war veterans with posttraumatic stress disorder: crosssectional study. Croat Med J 2007, 48:198-206

35. Davidson JR, Hughes D, Blazer DG, George LK: Post-traumatic stress disorder in the community: an epidemiological study. Psychol Med 1991, 21:713-721.

36. Kessler RC, Sonnega A, Bromet E, Hughes M, Nelson CB: Posttraumatic stress disorder in the National Comorbidity Survey. Arch Gen Psychiatry 1995, 52:1048-1060.

37. Matsuoka T, Yoshioka T, Oda J, Tanaka H, Kuwagata Y, Sugimoto H, Sugimoto T: The impact of a catastrophic earthquake on morbidity rates for various illnesses. Public Health 2000, 114:249-253.

38. Miller AC, Arquilla B: Chronic diseases and natural hazards: impact of disasters on diabetic, renal, and cardiac patients. Prehosp Disaster Med 2008, 23:185-194.

39. Jhung MA, Shehab N, Rohr-Allegrini C, Pollock DA, Sanchez R, Guerra F, Jernigan DB: Chronic disease and disasters medication demands of Hurricane Katrina evacuees. Am J Prev Med 2007, 33:207-210.

40. Toyabe S, Shioiri T, Kuwabara H, Endoh T, Tanabe N, Someya T, Akazawa K: Impaired psychological recovery in the elderly after the NiigataChuetsu Earthquake in Japan:a population-based study. BMC Public Health 2006, 6:230.

41. Fichter MM, Kohlboeck G, Quadflieg N: The Upper Bavarian longitudinal community study 1975-2004. 2. Long-term course and outcome of depression. A controlled study. Eur Arch Psychiatry Clin Neurosci 2008 258:476-488.

42. Yule W: Posttraumatic stress disorder in the general population and in children. J Clin Psychiatry 2001, 62(Suppl 17):23-28.

43. Pirkola S, Sund R, Sailas E, Wahlbeck K: Community mental-health services and suicide rate in Finland: a nationwide small-area analysis. Lancet 2009, 373:147-153.

44. Rust G, Daniels E, Satcher D, Bacon J, Strothers H, Bornemann T: Ability of community health centers to obtain mental health services for uninsured patients. JAMA 2005, 293:554-556.

\section{Pre-publication history}

The pre-publication history for this paper can be accessed here: http://www.biomedcentral.com/1471-2458/10/172/prepub

\section{doi: 10.1186/1471-2458-10-172}

Cite this article as: Jia et al., Are the elderly more vulnerable to psychological impact of natural disaster? A population-based survey of adult survivors of the 2008 Sichuan earthquake BMC Public Health 2010, 10:172

\section{Submit your next manuscript to BioMed Central and take full advantage of:}

- Convenient online submission

- Thorough peer review

- No space constraints or color figure charges

- Immediate publication on acceptance

- Inclusion in PubMed, CAS, Scopus and Google Scholar

- Research which is freely available for redistribution
C Biomed Central 